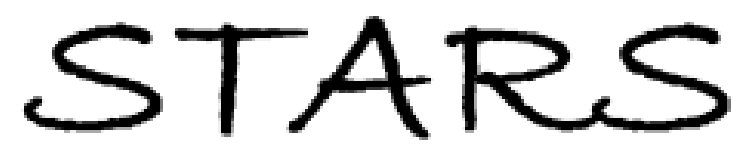

University of Central Florida

STARS

$1-1-2009$

\title{
Experimental observation of mode-selective anticrossing in surface-plasmon-coupled metal nanoparticle arrays
}

Amitabh Ghoshal

University of Central Florida

Ivan Divliansky

University of Central Florida

Pieter G. Kik

University of Central Florida

Find similar works at: https://stars.library.ucf.edu/facultybib2000

University of Central Florida Libraries http://library.ucf.edu

This Article is brought to you for free and open access by the Faculty Bibliography at STARS. It has been accepted for inclusion in Faculty Bibliography 2000s by an authorized administrator of STARS. For more information, please contactSTARS@ucf.edu.

\section{Recommended Citation}

Ghoshal, Amitabh; Divliansky, Ivan; and Kik, Pieter G., "Experimental observation of mode-selective anticrossing in surface-plasmon-coupled metal nanoparticle arrays" (2009). Faculty Bibliography 2000s. 1569.

https://stars.library.ucf.edu/facultybib2000/1569

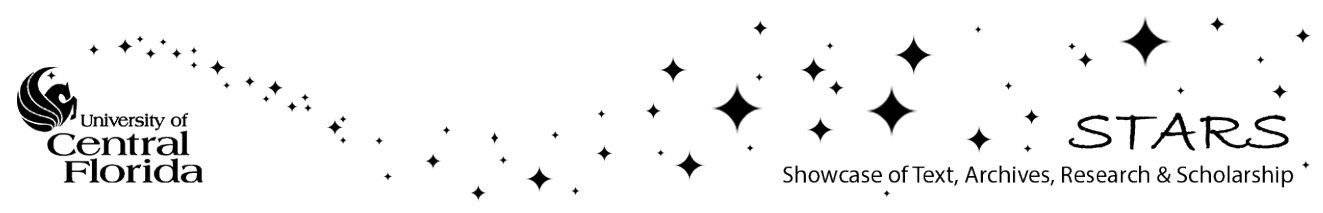




\section{Experimental observation of mode- selective anticrossing in surface-plasmon- coupled metal nanoparticle arrays}

Cite as: Appl. Phys. Lett. 94, 171108 (2009); https://doi.org/10.1063/1.3122922

Submitted: 19 March 2009 . Accepted: 31 March 2009 . Published Online: 29 April 2009

Amitabh Ghoshal, Ivan Divliansky, and Pieter G. Kik

\section{ARTICLES YOU MAY BE INTERESTED IN}

Theory and simulation of surface plasmon excitation using resonant metal nanoparticle arrays

Journal of Applied Physics 103, 113111 (2008); https://doi.org/10.1063/1.2936971

Experimental observation of narrow surface plasmon resonances in gold nanoparticle arrays

Applied Physics Letters 93, 181108 (2008); https://doi.org/10.1063/1.3012365

Plasmonics: Localization and guiding of electromagnetic energy in metal/dielectric structures

Journal of Applied Physics 98, 011101 (2005); https://doi.org/10.1063/1.1951057

\section{Applied Physics Letters}

Mid-IR and $\mathrm{THz}$ frequency combs special collection 


\title{
Experimental observation of mode-selective anticrossing in surface-plasmon-coupled metal nanoparticle arrays
}

\author{
Amitabh Ghoshal, ${ }^{\text {a) }}$ Ivan Divliansky, and Pieter G. Kik ${ }^{\text {b) }}$ \\ CREOL, The College of Optics and Photonics, University of Central Florida, 4000 Central Florida Blvd., \\ Orlando, Florida 32816, USA
}

(Received 19 March 2009; accepted 31 March 2009; published online 29 April 2009)

\begin{abstract}
Surface plasmon excitation using resonant metal nanoparticles is studied experimentally. Geometry dependent reflection measurements reveal the existence of several optical resonances. Strong coupling of the in-plane nanoparticle plasmon resonance and propagating plasmons is evident from clear anticrossing behavior. Reflection measurements at high numerical aperture demonstrate the excitation of surface plasmons via out-of-plane particle polarization. The thus excited plasmons do not exhibit anticrossing in the considered frequency range. The results are explained in terms of the known surface plasmon dispersion relation and the anisotropic frequency dependent nanoparticle polarizability. These findings are important for applications utilizing surface-coupled nanoparticle plasmon resonances. (C) 2009 American Institute of Physics. [DOI: 10.1063/1.3122922]
\end{abstract}

The field of integrated surface plasmon (SP) nanophotonics continues to develop rapidly due to the considerable advantages of SPs over traditional integrated photonic structures. SPs are charge density waves propagating on the interface between a metal and a dielectric, 1 and the high degree of confinement of SPs at a single interface allows for various easily fabricated single-interface waveguide designs. ${ }^{2-5} \mathrm{Di}-$ electric loaded SP waveguides ${ }^{2}$ represent a promising approach, allowing the development of SP-based optical links on standard silicon integrated circuits without the need for a thick lower cladding layer. In experimental studies, SP waveguides are often excited via methods analogous to prism coupling. ${ }^{1}$ Such approaches are not readily adapted to a standard integrated circuit fabrication process as they require macroscopic three-dimensional coupling elements. We have proposed the use of metal nanoparticle arrays for the efficient excitation of SPs using compact silicon-compatible nanostructures ${ }^{6}$ and have shown that the interaction between nanoparticle resonances and SPs in these arrays could lead to anticrossing. ${ }^{7}$ Recently, the existence of such anticrossing was demonstrated experimentally on particles arranged in a square lattice. ${ }^{8}$ In the present study, we investigate the excitation of SPs using dense arrays of anisotropic metal nanoparticles near a metal film. We investigate the interaction between the plasmon resonance of the nanoparticles and the grating resonance of the periodic structures using reflection spectroscopy. In addition to the predicted anticrossing between particle and grating resonance, we observe a second order mode as well as two additional modes not predicted by prior numerical simulations. Interestingly, the latter modes do not exhibit anticrossing in the entire observed frequency range. We show that these observations can be understood in terms of a mode-selective anticrossing caused by the anisotropic nature of the metal nanoparticle polarizability.

Samples were fabricated using multiple deposition and lithography steps. A $20 \mathrm{~nm}$ thick $\mathrm{Cr}$ adhesion layer was deposited on a Si substrate using thermal evaporation, followed by the deposition of a $200 \mathrm{~nm}$ thick gold layer. A $67 \mathrm{~nm} \mathrm{SiO} 2$

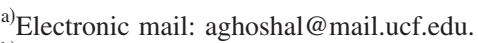

${ }^{b)}$ Also at the Physics Department, University of Central Florida.
}

spacer layer was deposited directly onto the gold layer by plasma-enhanced chemical vapor deposition. The $\mathrm{SiO}_{2}$ layer thickness was verified using spectroscopic ellipsometry. Gold nanoparticles were fabricated on the $\mathrm{SiO}_{2}$ layer using standard electron beam lithography patterning, followed by resist development and the subsequent deposition of a $5 \mathrm{~nm}$ $\mathrm{Cr}$ adhesion layer and a $34 \mathrm{~nm}$ thick Au layer. A schematic of the final sample structure obtained after lift-off is shown in Fig. 1 (inset). The nanoparticle dimensions along the $x, y$, and $z$ directions were 125,50 , and $39 \mathrm{~nm}$, respectively. Several particle arrays were fabricated, each consisting of 40 periods in the longitudinal $(x)$ and transverse $(y)$ directions, with a constant transverse center-to-center spacing of $L_{y}$ $=270 \mathrm{~nm}$ and a longitudinal center-to-center spacing $L_{x}$ between 505 and $1045 \mathrm{~nm}$.

Reflection spectra of the arrays were taken using an upright microscope. The sample was illuminated with a standard halogen light source through a $5 \times$ microscope

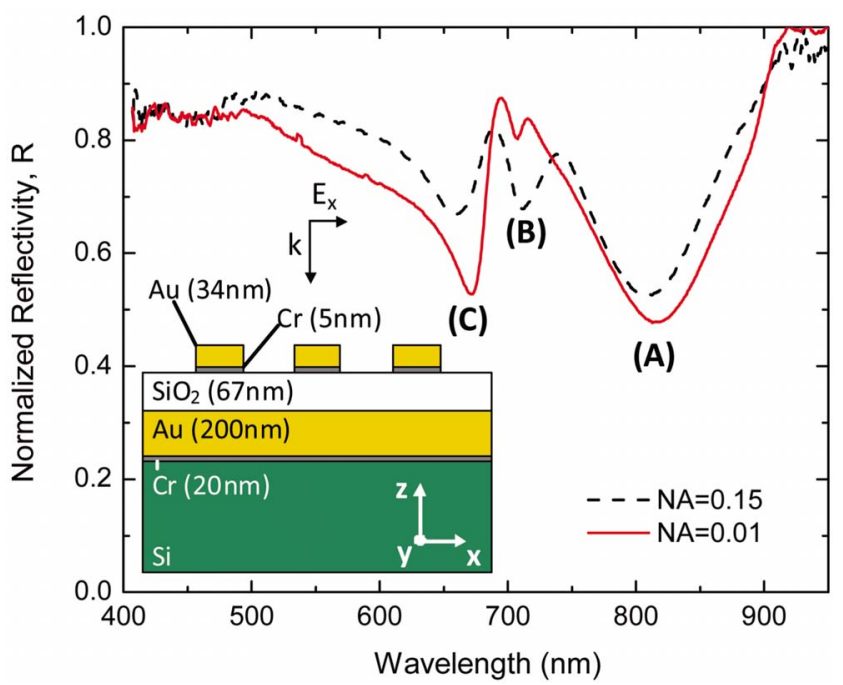

FIG. 1. (Color online) Normalized reflection spectra of a surface coupled nanoparticle array with longitudinal spacing $L_{x}=565 \mathrm{~nm}$ under two conditions: NA $=0.15$ (dashed line), and NA=0.01 (solid line). Inset: Schematic of the fabricated sample structure. 
objective. The incident light was polarized along the $x$ direction to within $10^{\circ}$ using a fixed polarizer in the illumination path. The numerical aperture (NA) of the illumination was varied between a low NA of 0.01 and a high NA of 0.15 by varying the aperture stop of the microscope. The sample was held perpendicular to the optical axis of the microscope to within $0.004^{\circ}$. For spectral analysis, the reflected light was directed to a multimode collection fiber with a core diameter of $50 \mu \mathrm{m}$ and sent to a single grating monochromator. Spectra were recorded using a thermoelectrically cooled silicon charge-coupled device camera attached to the monochromator. The integration time was adjusted to prevent signal saturation, and resulting spectra were averaged to improve the signal to noise ratio. For each measurement, an array was aligned with the collection area of the fiber and the signal $S(\lambda)$ was recorded as a function of wavelength $\lambda$. A reference spectrum $S_{\text {ref }}(\lambda)$ was taken by measuring the reflectivity of a nearby region without nanoparticles, and a dark spectrum $S_{\text {dark }}(\lambda)$ was also recorded. The normalized reflectivity $R$ was calculated as $R=\left(S-S_{\text {dark }}\right) /\left(S_{\text {ref }}-S_{\text {dark }}\right)$ and thus represents a change in reflectivity of the surface due to the presence of the periodic array. The spectral resolution in the measurements was approximately $4 \mathrm{~nm}$.

Figure 1 shows the reflection spectrum of an array with a longitudinal center-to-center spacing $L_{x}=565 \mathrm{~nm}$ recorded with a relatively high NA of 0.15 (dashed line). At this grating spacing constructive plasmon excitation is predicted to occur at a free space wavelength of $\lambda=685 \mathrm{~nm}$, based on the known layer thicknesses, $\mathrm{Au}$ dielectric function from literature, ${ }^{9}$ and measured $\mathrm{SiO}_{2}$ dielectric function. A pronounced reflection minimum (A) is observed at $810 \mathrm{~nm}$. This feature corresponds to the individual nanoparticle plasmon resonance for excitation with light polarized along the $x$ direction, as confirmed in independent measurements in which the particle aspect ratio was varied (not shown). Two additional reflection minima are observed at $\lambda \approx 660 \mathrm{~nm}$ and $\lambda$ $\approx 710 \mathrm{~nm}$, close to the predicted free space wavelength of propagating SPs if constructively excited by a grating of spacing $L_{x}=565 \mathrm{~nm}$. Upon reducing the illumination NA to 0.01 (solid line), the depth of the reflection minimum related to the particle resonance changes by only $8 \%$, while the depth of the two remaining reflection dips varies more strongly by $+22 \%$ and $-19 \%$ respectively. Since lowering the NA reduces the relative contribution of vertically polarized light, it appears that the reflection minimum at $710 \mathrm{~nm}$ (B) is related to a propagating SP grating mode excited by the $z$ polarization of the nanoparticles, while the minimum (C) at $660 \mathrm{~nm}$ is a grating resonance excited by the $x$ polarization of the nanoparticles.

Reflection spectra similar to those shown in Fig. 1 were taken at NA $=0.01$ for 19 different values of the longitudinal interparticle spacing $L_{x}$ from 505 to $1045 \mathrm{~nm}$ in steps of 30 $\mathrm{nm}$. Figure 2 shows the measured normalized reflectivity, with dark regions corresponding to low reflectivity. For the smallest grating spacing $\left(L_{x}=505 \mathrm{~nm}\right)$, the spectrum observed is similar to the low NA spectrum presented in Fig. 1. Two main minima at approximately 630 and $800 \mathrm{~nm}$ are observed (open circles), corresponding to the grating resonance and the $x$ polarization related particle resonance respectively. As the spacing $L_{x}$ is increased, the grating resonance is initially seen to redshift, and for large grating spacing $\left(L_{x}>800 \mathrm{~nm}\right)$ converges to the particle resonance

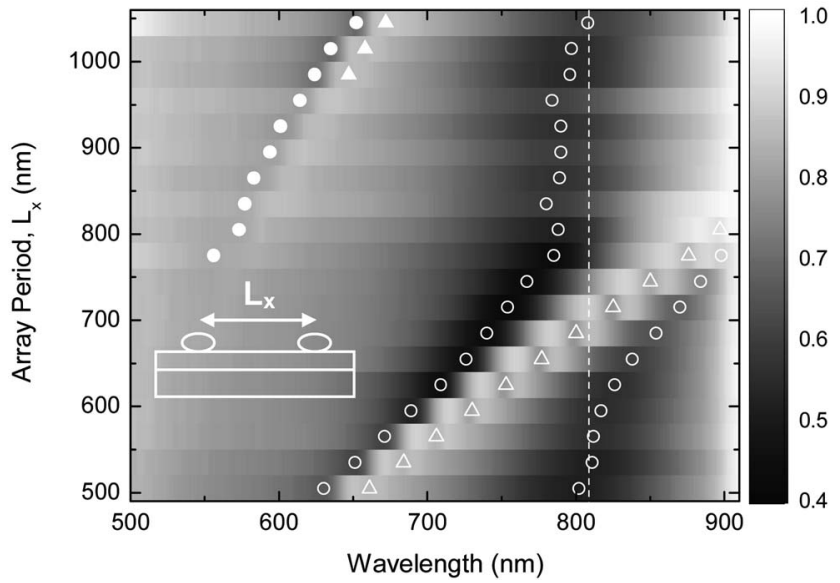

FIG. 2. Geometry dependent reflection spectra of surface-coupled metal nanoparticle arrays as a function of longitudinal particle spacing $L_{x}$. The color scale represents normalized reflectivity. Symbols mark the positions of reflection minima. The lateral nanoparticle resonance wavelength is indicated by the dashed line.

wavelength. The particle resonance on the other hand is initially independent of grating spacing but redshifts at grating spacing values $L_{x}>600 \mathrm{~nm}$. This pronounced anticrossing is due to the strong interaction of SP modes and the particle resonance, as predicted in simulations and discussed in detail in Ref. 7. Very different trends are observed for the weak resonance attributed to $z$-polarized excitation (open triangles). As $L_{x}$ is increased, the weak reflection minimum gradually redshifts with no discernible anticrossing at wavelengths close to the particle resonance. Above $L_{x}=805 \mathrm{~nm}$, an additional reflection minimum appears at $\lambda \approx 550 \mathrm{~nm}$ (filled circles) which redshifts as the grating spacing continues to increase. Above $L_{x}=955 \mathrm{~nm}$, a final reflection feature becomes discernable (filled triangles), which also redshifts for increasing grating spacing.

To understand the experimental observations, we compare the location of the various reflection minima to the SP dispersion relation. Figure 3 shows the calculated SP dispersion relation for the air-silica-gold system in the absence of nanoparticles, calculated using the method developed by Ward et al. ${ }^{10}$ as a function of SP wavevector $k_{x}=k_{\mathrm{SP}}$ using

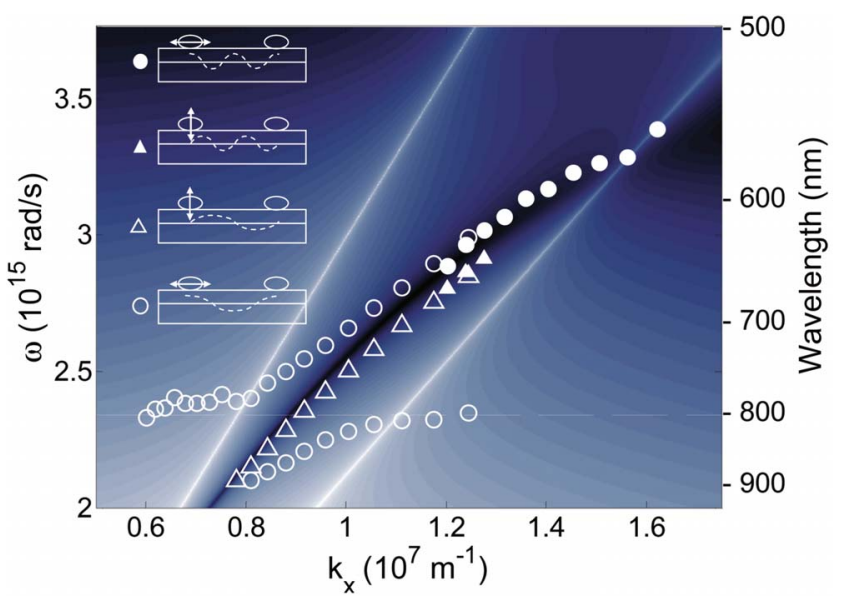

FIG. 3. (Color online) Calculated SP dispersion relation of the air-SiO $\mathrm{S}_{2}-\mathrm{Au}$ system. Experimentally observed reflection minima of the nanoparticle arrays are included as a function of $k_{x}=G$ (open symbols) and $k_{x}=2 G$ (filled symbols). The corresponding modes are indicated schematically. The lateral nanoparticle resonance frequency is indicated by the dashed line. 
literature values for the Au optical properties ${ }^{9}$ and measured values for the $\mathrm{SiO}_{2}$ thickness and optical properties. Allowed low-loss surface modes appear as dark narrow lines, and the left and right bright lines correspond to the air and silica light lines, respectively. In order to excite propagating SPs the grating equation needs to be satisfied, given by $k_{\mathrm{SP}}$ $=k_{\|} \pm n \times G$, where $k_{\|}$is the wavevector component of incident light projected along the surface, $G=2 \pi / L_{x}$ is the reciprocal grating vector of the structure, and $n=0,1,2 \ldots$ Under normal incidence illumination with low NA, we have $k_{\|} \approx 0$, leading to the excitation condition $k_{\mathrm{SP}}=G$ for $n=1$ (first order) and $k_{\mathrm{SP}}=2 G$ for $n=2$ (second order). The open symbols in Fig. 3 show the angular frequency of the three prominent reflection minima observed in Fig. 2 as a function of $k_{x}=G$. The weak reflection minima observed at $L_{x}>800 \mathrm{~nm}$ are shown as a function of $k_{x}=2 G$ (filled symbols). Several features should be noted. First, the main reflection minima (open circles) clearly exhibit anticrossing. At frequencies away from the anticrossing region at $\lambda \approx 800 \mathrm{~nm}$, the location of the main reflection minimum is seen to converge to the calculated SP dispersion relation. In the region of anticrossing, a large mismatch is observed between the positions of the reflection minima and the calculated SP dispersion relation, demonstrating that the large nanoparticle polarization near the particle resonance strongly affects the SP dispersion relation in the array. Note that the high-frequency reflection minima (filled symbols) shown as a function of $k_{x}=2 G$ also lie very close to the calculated dispersion relation, demonstrating that these features correspond to the excitation of plasmon modes with a SP wavelength that is approximately half the interparticle spacing.

Intriguingly, the weak reflection minima (open triangles) that were observed in Fig. 2 are seen to almost exactly follow the calculated dispersion relation in Fig. 3, without exhibiting any anticrossing near the nanoparticle resonance. This surprising result can be understood by considering the symmetry of the allowed modes. For near-normal incidence illumination, the $x$-polarized incident field excites an $x$-polarized plasmon resonance in the nanoparticles. The interaction of the propagating SPs with the strong $x$-polarized nanoparticle resonance gives rise to the anticrossing observed in Fig. 3, as also observed in numerical simulations ${ }^{7}$ and recent experimental work on square arrays of particles. ${ }^{8}$ At finite NA values, the convergent nature of the incident light introduces a field contribution polarized normal to the sample surface. This leads to a weak vertical polarization of the nanoparticles that also produces SP excitation. In principle, this interaction could lead to strong particle-SP coupling and anticrossing. However, due to the anisotropic particle shape (aspect ratio $\sim 2.5$ ), the vertical particle resonance occurs at much higher frequency than the lateral particle resonance. As a result, any effects related to interaction with a vertical nanoparticle resonance are expected to occur at frequencies well above those investigated here and, in fact, above frequencies at which propagating SPs can be excited in this material system. It is interesting to note that this vertically excited mode can act as an internal reference measurement, revealing the SP dispersion relation in the absence of strong particle-surface coupling. A final point worth noting is that the weak low-frequency second order $(n=2)$ features (solid triangles) overlap with the first order $(n=1)$ mode (open triangles) excited by vertical polarization, suggesting that these are also related to vertical polarization. The identification of all reflection features is summarized in the sketches in Fig. 3, where the dashed line indicates the strength of the $E_{x}$ electric field component along the $\mathrm{Au}-\mathrm{SiO}_{2}$ interface and the arrows indicate the corresponding particle polarization. The identification of all modes on this plasmon excitation structure is crucial for the further optimization of resonantly enhanced grating couplers, as the use of small coupler areas will necessitate the use of strongly convergent beams containing significant out-of-plane field components.

In summary, a spectroscopic analysis of electron-beam fabricated SP excitation structures was presented. Reflection measurements revealed various optical resonances that could all be identified by considering the SP dispersion relation and the anisotropic and frequency-dependent nanoparticle polarizability. The main reflection features were attributed to a lateral particle resonance and a SP related grating resonance. Varying the grating spacing revealed clear evidence of anticrossing, demonstrating strong coupling between the nanoparticle resonance and the grating resonance. An additional grating mode was found to be excited by incident field components normal to the sample surface and displayed no anticrossing due to the absence of a vertical particle plasmon resonance at the frequencies studied.

We would like to thank James Ginn for ellipsometry measurements and Dr. Min Hu for preparation of the reflection measurement setup. This work was supported by the National Science Foundation (CAREER Award No. ECCS0644228).

${ }^{1}$ H. Raether, Surface Plasmons on Smooth and Rough Surfaces and on Gratings, Springer Tracts in Modern Physics, Vol. 111 (Springer, Berlin, 1988).

${ }^{2}$ B. Steinberger, A. Hohenau, H. Ditlbacher, A. L. Stepanov, A. Drezet, F. R. Aussenegg, A. Leitner, and J. R. Krenn, Appl. Phys. Lett. 88, 094104 (2006).

${ }^{3}$ J. C. Weeber, Y. Lacroute, and A. Dereux, Phys. Rev. B 68, 115401 (2003).

${ }^{4}$ D. F. P. Pile, D. K. Gramotnev, M. Haraguchi, T. Okamoto, and M. Fukui, J. Appl. Phys. 100, 013101 (2006).

${ }^{5}$ K. Tanaka, M. Tanaka, and T. Sugiyama, Opt. Express 13, 256 (2005).

${ }^{6}$ A. Ghoshal, G. Webb-Wood, C. Mazuir, and P. G. Kik, Proc. SPIE 5927, 592714 (2005).

${ }^{7}$ A. Ghoshal and P. G. Kik, J. Appl. Phys. 103, 113111 (2008).

${ }^{8}$ Y. Chu and K. B. Crozier, Opt. Lett. 34, 244 (2009).

${ }^{9}$ P. B. Johnson and R. W. Christy, Phys. Rev. B 6, 4370 (1972).

${ }^{10}$ C. A. Ward, K. Bhasin, R. J. Bell, R. W. Alexander, and I. Tyler, J. Chem. Phys. 62, 1674 (1975). 